\title{
CCL2 released at tumoral level contributes to the hyperalgesia evoked by intratibial inoculation of NCTC 2472 but not B16-F10 cells in mice
}

\author{
Marta Pevida • Sara González-Rodríguez • Ana Lastra • \\ Agustín Hidalgo • Luis Menéndez • Ana Baamonde
}

Received: 30 March 2012 / Accepted: 18 July 2012 /Published online: 14 September 2012

(C) Springer-Verlag 2012

\begin{abstract}
The participation of the chemokine CCL2 (monocyte chemoattractant protein-1) in inflammatory and neuropathic pain is well established. Furthermore, the release of CCL2 from a NCTC 2472 cells-evoked tumor and its involvement in the upregulation of calcium channel $\alpha 2 \delta 1$ subunit of nociceptors was demonstrated. In the present experiments, we have tried to determine whether the increase in CCL2 levels is a common property of painful tumors and, in consequence, the administration of a chemokine receptor type 2 (CCR2) antagonist can inhibit tumoral hypernociception. CCL2 levels were measured by ELISA in the tumoral region of mice intratibially inoculated with NCTC 2472 or B16-F10 cells, and the antihyperalgesic and antiallodynic effects evoked by the administration of the selective CCR2 antagonist RS 504393 were assessed. Cultured NCTC 2472 cells release CCL2 and their intratibial inoculation evokes the development of a tumor in which CCL2 levels are increased. Moreover, the systemic or peritumoral administration of RS 504393 inhibited thermal and mechanical hyperalgesia, but not mechanical allodynia evoked after the inoculation of these cells. Thermal hyperalgesia was also inhibited by the peritumoral administration of a neutralizing CCL2 antibody. In contrast, no change in CCL2 levels was observed in mice inoculated with B16-F10 cells, and RS 504393 did not inhibit the hypernociceptive reactions
\end{abstract}

\footnotetext{
M. Pevida $\cdot$ S. González-Rodríguez • A. Lastra • A. Hidalgo • L. Menéndez $\cdot$ A. Baamonde $(\bowtie)$

Laboratorio de Farmacología. Facultad de Medicina. Instituto Universitario de Oncología del Principado de Asturias (IUOPA), Universidad de Oviedo,

c/ Julián Clavería 6,

33006 Oviedo, Asturias, Spain

e-mail: arbaiza@uniovi.es
}

evoked by their intratibial inoculation. The peripheral release of CCL2 is involved in the development of thermal and mechanical hyperalgesia, but not mechanical allodynia evoked by the inoculation of NCTC 2472 cells, whereas this chemokine seems unrelated to the hypernociception induced by B16-F10 cells.

Keywords Bone cancer-induced pain · Hyperalgesia . Allodynia $\cdot$ CCL2 $\cdot$ CCR2 antagonists · Mouse · NCTC 2472 cells

\section{Introduction}

CCL2, also called monocyte chemoattractant protein-1 (MCP-1), is a member of the CC group of chemokines that, apart from its involvement in monocyte/macrophage chemotaxis, plays an important role in the modulation of nociception in pathological settings (Abbadie et al. 2009). As occurs with the majority of chemokines, CCL2 does not act exclusively on a unique type of receptors, its main effects being evoked through the activation of chemokine receptor type 2 (CCR2) (Abbadie et al. 2003).

Different experimental data illustrate a complex modulation of nociceptive processing by the CCL2/CCR2 system both at peripheral and spinal level. Thus, the ability of CCL2 to provoke neural sensitization in the spinal cord has been demonstrated by electrophysiological recordings of spinal lamina II neurons (Gao et al. 2009) and also by behavioral experiments in which hypernociception occurs when CCL2 is directly administered into the spinal cord of rodents (Tanaka et al. 2004; Dansereau et al. 2008; Baamonde et al. 2011). 
More directly related with the present experiments is the involvement of CCL2 in the peripheral processing of nociceptive signals. A role for CCL2 as peripheral sensitizer is supported by the induction of mechanical (Bogen et al. 2009) and thermal (Qin et al. 2005) hyperalgesia following its administration into the rat paw. CCL2 can sensitize nociceptors through several mechanisms, such as the stimulation of phospholipase $\mathrm{C}$ and protein kinase $\mathrm{C}$ together with the release of calcium (Qin et al. 2005), the transactivation of capsaicin-sensitive transient receptor potential vanilloid 1 (TRPV1) (Jung et al. 2008), or the enhancement of Nav1.8 channels excitability (Belkouch et al. 2011). Interestingly, CCL2-inmunoreactive inflammatory cells are detected in inflamed tissues (Ogura et al. 2010), and the increased presence of this chemokine has also been demonstrated in dorsal root ganglia (DRG) of rats inflamed with complete Freund's adjuvant (Jeon et al. 2008). Supporting the relevance of these findings, a possible correlation between CCL2 gene expression in inflamed tissue and pain intensity perceived in patients with oral inflammation has been proposed (Wang et al. 2009). Besides, CCL2 also plays a prominent role in the reaction that appears after nerve injury. Both CCL2 and CCR2 can be upregulated at DRG neurons in neuropathic settings (White et al. 2005; Jeon et al. 2011), and it has been further described that CCL2 upregulation seems to be related to the presence of TNF $\alpha$ (Jeon et al. 2011) or IL-1 $\beta$ (White et al. 2005). In accordance with these studies, mice lacking CCR2 show decreased nociceptive behaviors after neuropathic injury (Abbadie et al. 2003).

Our knowledge related to the involvement of CCL2 in painful neoplastic processes is more limited. It seems likely that CCL2 could participate in tumoral hypernociception since its release has been detected from different types of human tumors, some of which are associated with painful symptoms such as pancreatic cancer (Chehl et al. 2009) or bone metastases derived from prostate or breast neoplastic processes (Shirotake et al. 2012; Soria et al. 2011). Experimentally, Khasabova et al. (2007) have demonstrated an increase in CCL2 concentrations at tumoral level in mice inoculated with NCTC 2472 fibrosarcoma cells into the calcaneus. In these studies, the participation of CCL2 in the induction of phenotypic changes that can lead to hyperalgesia, such as the increase in the expression of $\alpha_{2} \delta-1$ subunit of calcium channels in nociceptors, was also shown (Khasabova et al. 2007). Although these data seem to indicate that CCL2 could play a role in the development of tumoral hyperalgesia, the possible antihyperalgesic effect evoked by CCR2 antagonists has not been assessed in experimental models of neoplastic hypernociception. Thus, in the present experiments, we study whether CCL2 concentrations are increased at tumoral level and DRG in two models of murine bone cancer-induced pain. The intratibial inoculation of NCTC fibrosarcoma 2472 cells in $\mathrm{C} 3 \mathrm{H} / \mathrm{He}$ mice induces osteolytic lesions accompanied by thermal and mechanical hyperalgesia as well as mechanical allodynia that can be measured at weeks 4 and 2, respectively (Menéndez et al. 2003: Baamonde et al. 2007). Besides, the inoculation of B16-F 10 melanoma cells induces rapid changes in bone (osteoid formation with osteolytic lesions) together with thermal and mechanical hyperalgesia as well as mechanical allodynia during the first week after their inoculation (Curto-Reyes et al. 2008). In these tumor-bearing mice, we further explored whether the administration of RS 504393, a selective CCR2 antagonist, can inhibit bone cancer-evoked hypernociception.

\section{Methods}

Animals

Experiments were performed in 5- to 6-week-old $\mathrm{C} 3 \mathrm{H} / \mathrm{He}$ and C57BL/6 male mice bred in the Animalario de la Universidad de Oviedo (Reg. 33044 13A), maintained on a 12-h dark-light cycle with free access to food and water. Experimental procedures were approved by the Comité Ético de Experimentación Animal de la Universidad de Oviedo (Asturias, Spain) and according to the guidelines for the treatment of animals of the International Association for the Study of Pain (Zimmermann 1983). Each animal was used only once. Behavioral experiments were performed between 15:00 and 20:00 in a thermostated $\left(21^{\circ} \mathrm{C}\right)$ and noise-isolated room. The experimenter was not systematically unaware about the treatment received by each mouse. In order to minimize experimental bias, mice were randomly assigned to solvent or drug treatment, taking care that animals treated with solvent and with different doses of drug were tested in every session.

\section{Cell inoculation}

NCTC 2472 cells (American Type Culture Collection, ATCC) were cultured in NCTC 135 medium (Sigma) containing $10 \%$ horse serum (Sigma), passaged weekly according to ATCC guidelines. At confluence, cells were detached by scraping, centrifuged at $400 \times \mathrm{g}$ for $10 \mathrm{~min}$ and the remaining pellet suspended in PBS (Menéndez et al. 2003). B16-F10 melanoma cells (American Type Culture Collection) were cultured in DMEM (Gibco) enriched with $10 \%$ fetal calf serum (FCS, Gibco). When cells were preconfluent, they were treated with trypsin/EDTA (0.05/0.02\%) and detached. The trypsin/EDTA solution was recovered, neutralized with DMEM supplemented with $10 \%$ FCS and centrifuged at $400 \times \mathrm{g}$ for 10 min (Curto-Reyes et al. 2008).

For surgical procedures, anesthesia was induced by spontaneous inhalation of $3 \%$ isoflurane (Isoflo ${ }^{\circledR}$, Esteve) and maintained by administering $1.5 \%$ isoflurane in oxygen through a breathing mask. A suspension of $10^{5}$ cells in 
$5 \mu \mathrm{l}$ of PBS was injected into the right tibial medullar cavity, and after applying acrylic glue (Hystoacril ${ }^{\circledR}$, Braun) on the tibial plateau incised area, surgery was finished with a stitch of the skin. Control mice received the inoculation of $10^{5}$ cells previously killed by quickly freezing them three times without cryoprotection. According to the appropriate timing to reach maximal hypernociception, mechanical allodynia was assessed 2 weeks after the inoculation of NCTC 2472 cells and thermal and mechanical hyperalgesia 4 weeks after cell implantation (Menéndez et al. 2003). In mice that received intratibial B16-F10 cells, experiments were performed 1 week after inoculation (Curto-Reyes et al. 2008).

\section{Drugs}

The CCR2 receptor antagonist, RS 504393 (Tocris), or its corresponding solvent (5\% DMSO at the highest concentration used) was subcutaneously administered under the fur of the neck in a volume of $10 \mathrm{ml} / \mathrm{kg} 30 \mathrm{~min}$ before testing.

RS 504393 or its solvent, a goat anti-mouse CCL2 antibody (R\&D, AF-479-NA) or an anti-mouse goat antiserum diluted in saline at the same IgG concentration than the anti-CCL2 antibody (Sigma) were peritumorally administered. The crossreactivity of this antibody against other mouse chemokines such as MCP-5, MIP $1 \alpha$, MIP $1 \beta$, MIP $1 \gamma$, MIP-2, MIP $3 \beta$, and RANTES is less than $0.01 \%$. For peritumoral administration, drugs were injected subcutaneously over the tibial tumor mass in a volume of $100 \mu 1$. Injections in the left, contralateral, paw were performed in the same region of the limb which was, in this case, free of tumor.

\section{Behavioral studies}

\section{Unilateral hot plate test}

Thermal withdrawal latencies were measured by the unilateral hot plate test (Menéndez et al. 2003). Briefly, mice were gently restrained and the plantar side of the tested paw placed on the hot plate surface $\left(50.5^{\circ} \mathrm{C}\right.$ for $\mathrm{C} 3 \mathrm{H} / \mathrm{He}$ mice and $49.5^{\circ} \mathrm{C}$ for B16-F10) as previously described (Curto-Reyes et al. 2010). Measurements of withdrawal latencies from the heated surface of each hind paw were made separately at 2-min intervals and the mean of two measures was considered. A cut-off of $20 \mathrm{~s}$ was established.

\section{Paw pressure test}

Mechanical withdrawal latencies were measured by a previously described adaptation of the Randall-Selitto method (Menéndez et al. 2005), in which a constant pressure stimulus is used. Mice were gently restrained and a pressure of $450 \mathrm{~g}$ was applied to their hindpaws with a Ugo Basile 7200 apparatus until a struggle reaction appears. The measurements of the withdrawal latencies of each hindpaw were made separately and alternately at 2-min intervals and the mean of two measures made in each hindpaw was considered. A 60 -s cutoff was established in order to prevent tissue damage.

von Frey test

Mechanical allodynia was assessed by applying von Frey filaments (Stoelting) to the plantar side of the paws as previously reported (Baamonde et al. 2007). Mice were placed on a wire mesh platform and allowed for habituation for $20 \mathrm{~min}$. The von Frey filaments 2.44, 2.83, 3.22, 3.61, 4.08, and 4.56 were used, and, starting with the 3.61 filament, six measurements were taken in each animal randomly starting by the left or right paw. Based on the "up and down" method (Chaplan et al. 1994), the observation of a positive response (lifting, shaking, or licking of the paw) was followed by the application of the immediate thinner filament or the immediate thicker one if the response was negative. The $50 \%$ response threshold was calculated using the following formula: $50 \% \mathrm{~g}$ threshold $=$ $\left(10^{\mathrm{Xf}+\kappa \delta}\right) / 10,000$; where $\mathrm{Xf}$ is the value of the last von Frey filament applied; $\kappa$ is a correction factor based on pattern of responses (from the Dixon's calibration table); and $\delta$ is the mean distance in log units between stimuli (here, 0.4).

\section{Enzyme-linked immunosorbent assay (ELISA)}

CCL2 levels were measured in NCTC 2472 and B16-F10 cell culture medium before and after the incubation of cells during 4 days in flasks with $15 \mathrm{ml}$ when cells reached confluence. Experiments were also performed in tissue homogenates prepared from hind limbs or DRG either ipsilateral or contralateral to the inoculated paw of mice receiving live or killed tumoral cells. Once harvested, media and tissues were immediately frozen in liquid nitrogen and stored at $-80^{\circ} \mathrm{C}$ until use.

Hind limbs were denuded from ankle to knee, including bone and soft tissue, and individually homogenized. Homogenates of DRG were prepared in pools of L3-L5 DRG obtained from six animals (18 DRG per homogenate). The buffer used consisted $0.1 \mathrm{M}$ Tris, $0.15 \mathrm{M} \mathrm{NaCl}, 0.5 \%$ CTAB (Fluka), and a protease inhibitor (1 tablet $/ 50 \mathrm{ml}$ buffer, Roche Diagnostics). Limbs were homogenized in a volume of $3 \mu \mathrm{l} / \mathrm{mg}$ with a Polytron PT-MR3100 (Kinematica) and DRG in a volume of $24 \mu \mathrm{l}$ by using a Minicraft MB130. Next, homogenates were centrifuged at $15,000 \times \mathrm{g}$ for $15 \mathrm{~min}$ at $4{ }^{\circ} \mathrm{C}$, and protein concentration of supernatants was measured by a BCA protein assay (Pierce) according to the manufacturer's protocol.

The level of CCL2 was measured with a commercially available sandwich enzyme-linked immunosorbent assay (R\&D Systems, DuoSet ${ }^{\circledR}$ Mouse CCL2/JE/MCP-1). Following the instructions of the manufacturer, plates (R\&D 
Systems) were coated overnight at room temperature with an antibody specific for mouse CCL2. In order to achieve a value in the range of the standard curve of CCL2, protein quantities added to the wells for the different homogenates were $100 \mu \mathrm{g}$ for limbs and $70 \mu \mathrm{g}$ for DRG in a final volume of $100 \mu \mathrm{l}$. After washing, a 2-h incubation period was performed with a second biotinylated anti-mouse CCL2 antibody and followed by a 20 min incubation period with streptavidin-peroxidase (HRP). After washing to remove all the unbound enzyme, color was developed by adding a stabilized chromogen (tetramethylbenzidine: $\left.\mathrm{H}_{2} \mathrm{O}_{2}, 1: 1\right)$ and the reaction was terminated with a stop solution $\left(2 \mathrm{~N} \mathrm{H}_{2} \mathrm{SO}_{4}\right.$ ). The intensity of the colored product was quantified spectrophotometrically at $450 \mathrm{~nm}$ substracting the readings obtained at $570 \mathrm{~nm}$ in order to correct optical background of plates.

Values obtained from culture media and limbs came from four to five independent measures performed in duplicate and in the case of DRG, from five independent data.

\section{Statistical analysis}

The mean values and the corresponding standard errors were calculated for each ELISA measurement or behavioral assay. The values of CCL2 levels obtained in culture media were compared by a Student's $t$ test and those obtained from tissue homogenates were compared by an initial one-way analysis of variance (ANOVA) followed by the Newman-Keuls test. Thermal and mechanical withdrawal latencies were compared by the Student's $t$ test for grouped values when the latency obtained in the ipsilateral paw was compared with that obtained in the contralateral one or by a one-way ANOVA followed by the Dunnett's $t$ - when groups that received different doses of RS 504393 were compared with the solvent-treated one. Threshold values obtained by the von Frey test in ipsilateral and contralateral paws were compared by the $U$ Mann-Whitney's test. In all cases, the level of significance was set at $P<0.05$.

\section{Results}

Measurement of CCL2 levels in the culture medium of NCTC 2472 cells and in samples coming from limbs and DRG of mice intratibially inoculated with NCTC 2472

In order to determine if tumoral cells can release CCL2, the concentration of this chemokine was measured by ELISA in the culture medium before and after NCTC 2472 cell incubation. Low CCL2 levels were present in the medium before its contact with tumoral cells, whereas an important increase (eight times) was found 4 days after culture when cells reached confluence (Fig. 1a).

In homogenates prepared from the tibial region obtained from ipsilateral and contralateral limbs of $\mathrm{C} 3 \mathrm{H} / \mathrm{He}$ mice
4 weeks after the intratibial inoculation of either live or killed NCTC 2472 cells, the levels of CCL2 were also measured. Similar low [CCL2] were obtained in homogenates prepared both from limbs of mice inoculated with killed cells and from the contralateral paw of osteosarcoma-bearing mice. In contrast, the concentrations measured in homogenates coming from tumor-bearing paws were about 12 times higher (Fig. 1b).

No change in the levels of CCL2 measured in homogenates prepared from L3-L5 DRG was detected when comparing those obtained in the ipsilateral side with those of the contralateral ones in mice inoculated with killed or live NCTC 2472 cells (Fig. 1c).

Measurement of CCL2 levels in the culture medium of B16-F10 cells and in samples coming from limbs and DRG of mice intratibially inoculated with B16-F10

No change was detected in the concentration of CCL2 detected in culture medium before and after the growth of B16-F10 cells (Fig. 2a). Also, similar CCL2 levels were found when homogenates of the tibial region of mice inoculated with killed or live B16-F10 cells 1 week before were compared (Fig. 2b). The values obtained in homogenates prepared with ipsilateral or contralateral DRG coming from mice inoculated with killed or live B16-F10 cells were also indistinguishable (Fig. 2c).

The administration of the CCR2 antagonist RS 504393 or a CCL2 antibody inhibits NCTC 2472-evoked thermal hyperalgesia

The intratibial inoculation of live NCTC 2472 cells 4 weeks before evoked a thermal hyperalgesic reaction. This thermal hyperalgesia was dose-dependently inhibited $30 \mathrm{~min}$ after the s.c. administration of the selective CCR2 antagonist RS $504393(0.3-3 \mathrm{mg} / \mathrm{kg})$. The dose of $0.3 \mathrm{mg} / \mathrm{kg}$ did not modify thermal withdrawal latencies, a partial antihyperalgesic effect was observed after the administration of $1 \mathrm{mg} /$ $\mathrm{kg}$, and a complete inhibition of osteosarcoma-induced hyperalgesia was attained when $3 \mathrm{mg} / \mathrm{kg}$ of RS 504393 was administered (Fig. 3a). The administration of the highest dose did not modify thermal latencies in mice inoculated with killed NCTC 2472 cells (data not shown).

In order to elucidate whether the analgesic effect evoked by RS 504393 in tumor-bearing mice could be peripherally mediated, RS 504393 was locally administered at tumoral level. A dose-dependent antihyperalgesic effect was again observed $30 \mathrm{~min}$ after the peritumoral injection of 3-30 $\mu \mathrm{g}$ of the CCR2 antagonist (Fig. 3b). In contrast, the administration of the maximal dose in the contralateral paw did not modify thermal withdrawal latencies in the tumor-bearing paws (not shown), supporting the involvement of locallyproduced antihyperalgesic effects. 


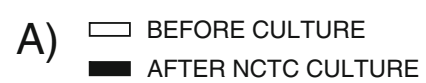

B)
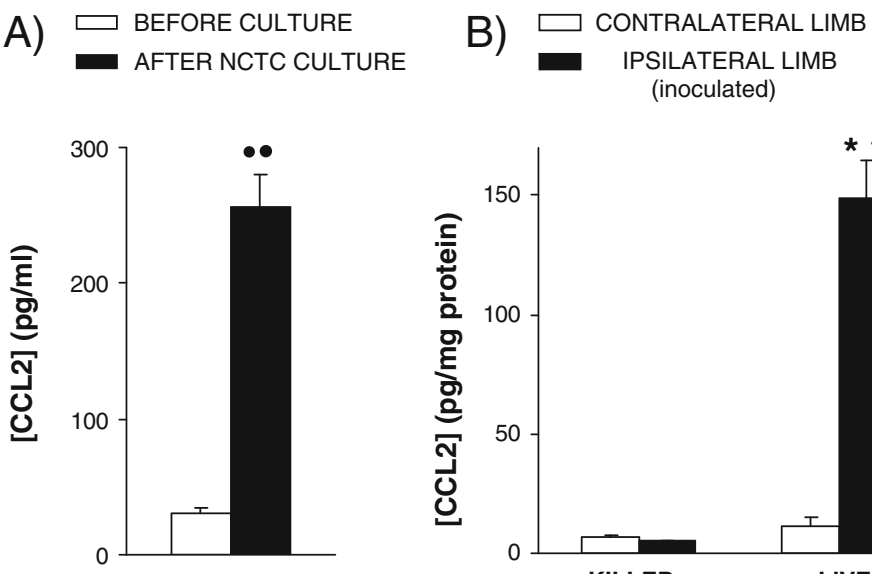

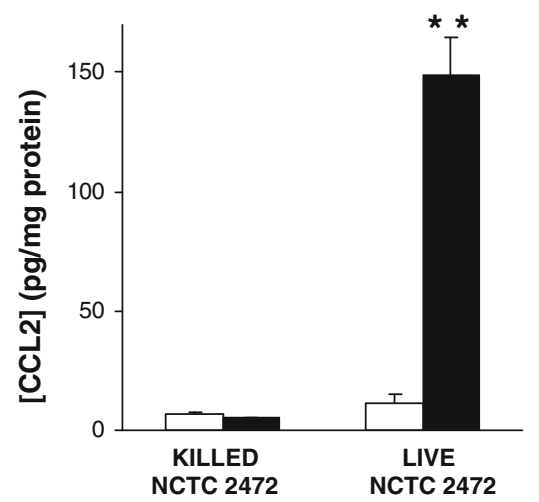

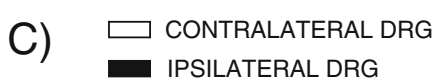

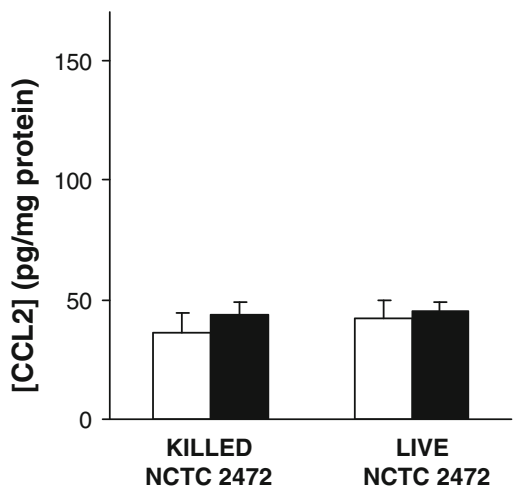

Fig. 1 CCL2 levels measured by ELISA. a [CCL2] in NCTC 2472 culture medium in the absence of cells and after cell confluence. $\mathbf{b}$ and c CCL2 levels measured in homogenates of hindpaws (b) or of L3-L5 DRG (c) of mice intratibially inoculated 4 weeks before with $10^{5}$ live or killed NCTC 2472 cells. Means and their corresponding standard

Since CCL2 is one of the main endogenous chemokines that bind CCR2, we have assessed the effect of neutralizing this chemokine. The peritumoral administration of a CCL2anti-mouse antibody $(4 \mu \mathrm{g})$ completely blocked thermal hyperalgesic responses induced in these tumor-bearing mice (Fig. 3c), supporting the role of this chemokine. The administration of this dose of antibody showed no effect in mice inoculated with killed NCTC 2472 cells (not shown).

The administration of the CCR2 antagonist RS 504393 inhibits mechanical hyperalgesia, but not mechanical allodynia, evoked by the inoculation of NCTC 2472 cells

A decrease in withdrawal latencies was measured in the paw pressure test 4 weeks after the intratibial inoculation of errors are represented $(n=4-5)$. Double black circles indicate $P<0.01$ compared with the values obtained before culture. Double asterisks indicate $P<0.01$ compared with contralateral limbs and limbs inoculated with killed cells, Newman-Keuls test

NCTC 2472 cells. This mechanical hyperalgesia was completely inhibited $30 \mathrm{~min}$ after the administration of RS 504393 either subcutaneously ( $3 \mathrm{mg} / \mathrm{kg}$; Fig. 4a) or peritumorally ( $30 \mu \mathrm{g}$; Fig. 4b). The administration of $3 \mathrm{mg} / \mathrm{kg}$ of RS 504393 to mice inoculated with killed NCTC 2472 or the administration of $30 \mu \mathrm{g}$ in the contralateral paw of tumor-bearing mice did not modify withdrawal latencies in the paw pressure test (data not shown).

Mechanical allodynia measured by the von Frey test appears in mice inoculated 2 weeks before with NCTC 2472 cells. The lowered threshold measures obtained in the von Frey test in inoculated mice were not modified by the s.c. administration of RS 504393 (3 mg/kg; Fig. 4c), thus mechanical allodynia being unaffected by the blockade of CCR2 receptors.

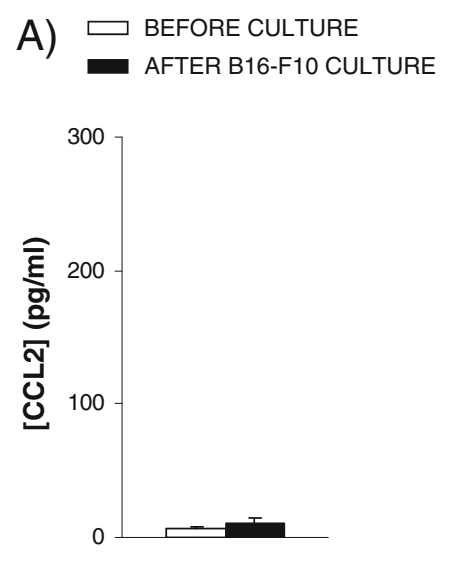

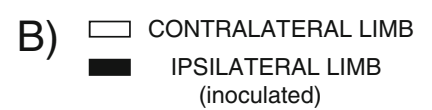

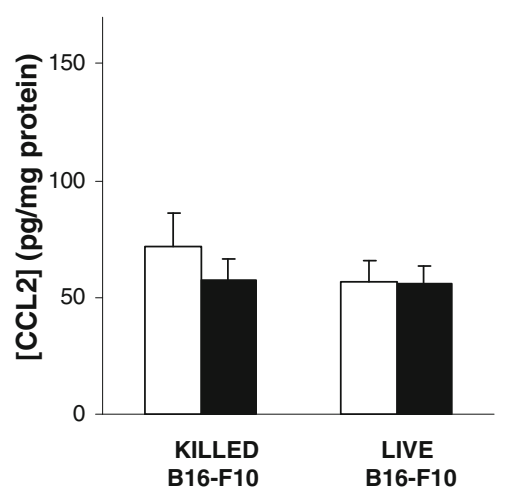

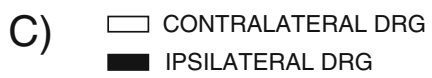

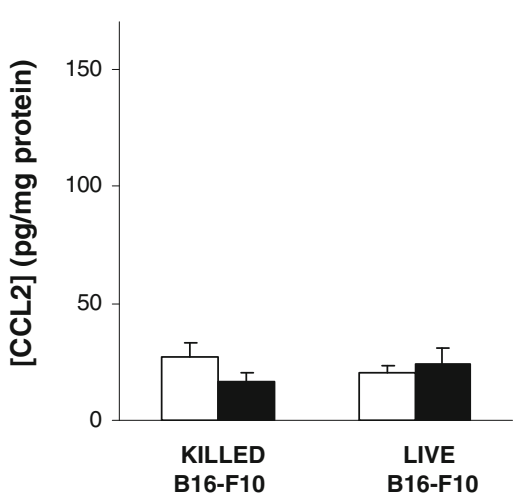

Fig. 2 CCL2 levels measured by ELISA. a [CCL2] in B16-F10 culture medium in the absence of cells and after cell confluence. b and c CCL2 levels measured in homogenates of hind paws (b) or of
L3-L5 DRG (c) of mice intratibially inoculated 4 weeks before with $10^{5}$ live or killed B16-F10. Means and their corresponding standard errors are represented $(n=4-5)$ 
A) UHP

B) UHP

C) UHP
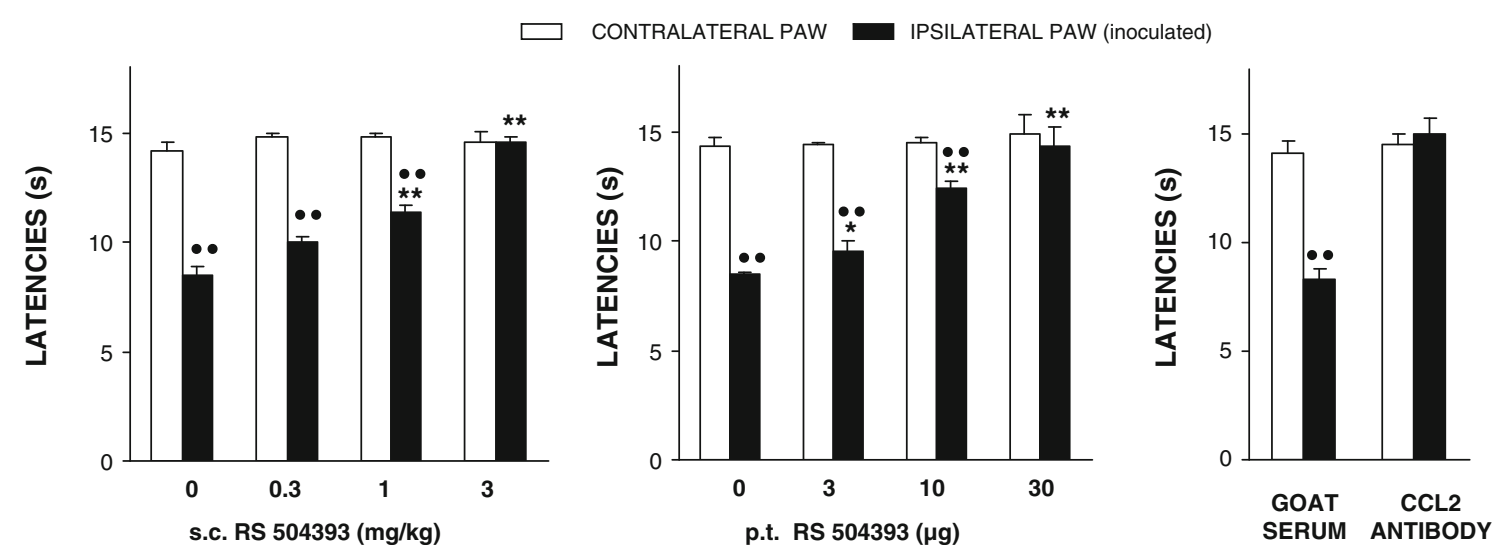

Fig. 3 Effects of RS 504393 or a CCL2 antibody on thermal withdrawal latencies measured in the unilateral hot plate test $(U H P)$ in mice intratibially inoculated with live NCTC 2472 cells. In a, RS 504393 $(0-3 \mathrm{mg} / \mathrm{kg}$ ) was subcutaneously (s.c.) administered $30 \mathrm{~min}$ before. In b and c, RS $504393(3-30 \mu \mathrm{g})$ or the CCL2 antibody $(4 \mu \mathrm{g})$ were peritumorally (p.t.) administered into the inoculated paw $30 \mathrm{~min}$

before. Means and corresponding standard errors are represented $(n=$ 5-6). Double black circles indicate $P<0.01$ compared with its corresponding, contralateral, left paw, Student's $t$ test; Single asterisk indicates $P<0.05$; double asterisks indicate $P<0.01$ compared with solvent-treated group, Dunnett's $t$ test

The administration of the CCR2 antagonist RS 504393 does not modify the hyperalgesia or allodynia evoked by the inoculation of B16-F10 cells

Thermal and mechanical hyperalgesia as well as mechanical allodynia can be measured in mice inoculated 1 week before with B16-F10 cells. A slight inhibition of thermal hyperalgesia appeared after the administration of the maximal dose of RS 504393 assayed subcutaneously $(3 \mathrm{mg} / \mathrm{kg}$ ), remaining withdrawal latency values significantly lower than those obtained in the contralateral paw (Fig. 5a). A similar result was obtained

after the local administration of RS 504393 over the tumoral mass since only the maximal dose assayed $(30 \mu \mathrm{g})$ induced an antihyperalgesic effect although withdrawal latencies remained significantly lower than those obtained in the contralateral noninjured paw (Fig. 5b). As shown in Figs. 5c and d, the low mechanical withdrawal latencies and mechanical threshold values obtained in tumor-bearing paws remained unaffected after the systemic administration of RS $504393(3 \mathrm{mg} / \mathrm{kg})$ $30 \mathrm{~min}$ before. In all cases, thermal and mechanical latencies as well as mechanical thresholds remained unaltered in mice inoculated with B16-F10 killed cells (data not shown).
A) PPT
B) PPT
C) $\mathrm{VF}$

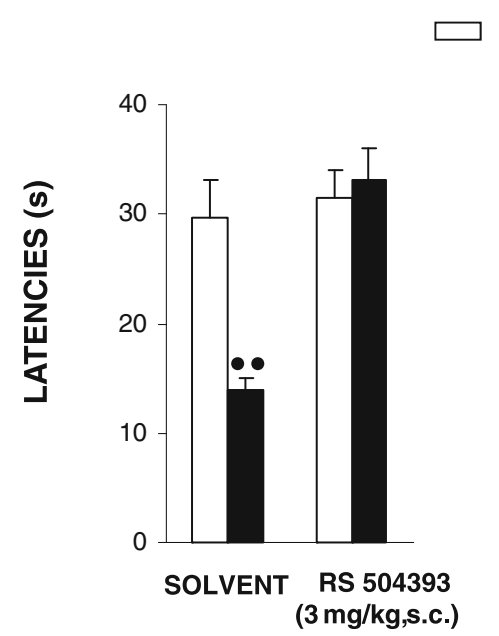

CONTRALATERAL PAW

IPSILATERAL PAW (inoculated)
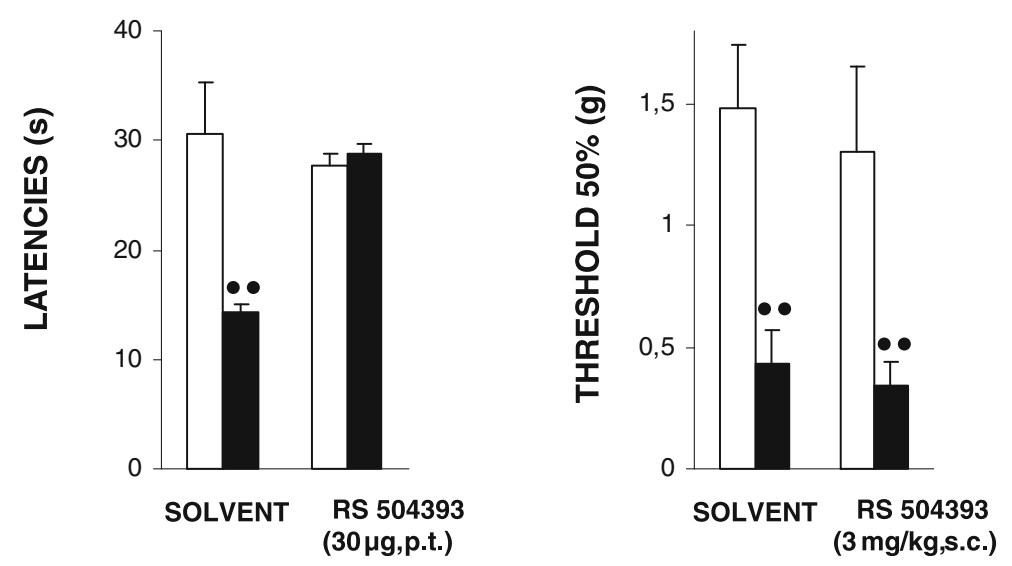

Fig. 4 Effects of RS 504393 in the paw pressure test $(P P T)$ and von Frey $(V F)$ test in mice intratibially inoculated with live NCTC 2472 cells. Inhibition of mechanical hyperalgesia after subcutaneous (s.c. $3 \mathrm{mg} / \mathrm{kg}$, a) or peritumoral (p.t. $30 \mu \mathrm{g}, \mathbf{b}$ ) administration of RS 504393. c Absence of

antiallodynic effect after the s.c. administration of RS $504393(3 \mathrm{mg} / \mathrm{kg}$, c). Means and corresponding standard errors are represented $(n=5-7)$. Double black circles indicate $P<0.01$ compared with its corresponding left paw, Student's $t$ test $(\mathbf{a}, \mathbf{b})$ or Mann-Whitney's $U$ test (c) 
A) UHP

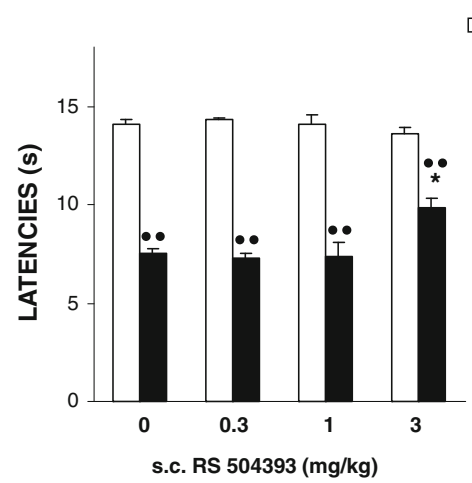

B) UHP

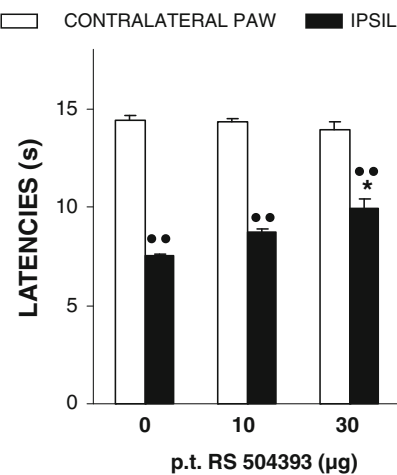

C) PPT

D) $\mathrm{VF}$
Fig. 5 Effects of RS 504393 in mice intratibially inoculated with live B16-F10 cells. In a and b RS 504393 was tested in the unilateral hot plate test $(U H P)$ after its subcutaneous (s.c.; $0-3 \mathrm{mg} / \mathrm{kg}, \mathrm{A}$ ) or peritumoral (p.t., 10 and $30 \mu \mathrm{g}, \mathbf{b}$ ) administration. In $\mathbf{c}$ and $\mathbf{d}$, the effects produced by RS 504393 in the paw pressure test $(P P T)$ and von Frey $(V F)$ tests after its

\section{Discussion}

The present study shows that the chemokine CCL2 can be released in particular bone cancer-induced pain processes in which CCR2 receptor antagonists can exert an antihyperalgesic effect. Our results confirm previous data showing that CCL2 is released from NCTC 2472 cells in culture (Khasabova et al. 2007; Schiller et al. 2009) and in vivo from the osteosarcoma developed after their intraosteal inoculation (Khasabova et al. 2007). Moreover, behavioral results obtained after the administration of the CCR2 antagonist RS 504393 demonstrate that CCL2 released at tumoral level plays a hyperalgesic role in osteosarcoma-bearing mice. In contrast, the measurement of CCL2 released from melanoma B16-F10 cells in culture as well as from tumors due to their intratibial inoculation indicates that the production of CCL2 by these cells is under detection limits either in vitro or in vivo, and, accordingly, the administration of RS 504393 is almost ineffective in this case.

Initially, the results obtained with NCTC 2472 cells ratify that these cells can release CCL2 in culture and that this property is maintained in vivo after their inoculation into the medullar cavity of the tibia since CCL2 levels are also augmented in tumoral tissue. As increasingly characterized, a cross-talk exists in the injured tissue, between pronociceptive cytokines, peptides, and modulators derived from tumoral and immune cells, as well as from osteoclasts (Stösser et al. 2011). In fact, it has been demonstrated that the release of CCL2 by NCTC 2472 cells is potentiated when these tumoral cells are cocultured with bone, especially if its hematopoietic system is conserved (Schiller et al. 2009). Furthermore, particular mediators involved in the nociceptive reactions measured in this bone cancer-induced model of pain could also contribute to the release of CCL2. This could be the case for the hematopoietic-released factors (Schweizerhof et al. 2009) that evoke the release of CCL2 in other types of tumors (Owen et subcutaneous administration $(3 \mathrm{mg} / \mathrm{kg}$ ) are shown. Means and corresponding standard errors are represented $(n=5-7)$. Double black circles indicate $P<0.01$ compared with its corresponding left paw, Student's $t$ test $(\mathbf{a}, \mathbf{b}, \mathbf{c})$ or Mann-Whitney's $U$ test $(\mathbf{d})$; double asterisks indicate $P<0.01$ compared with solvent-treated group, Dunnett's $t$ test

al. 2007), IL-1 $\beta$ (Baamonde et al. 2007) whose presence can activate the CCL2 gene in synoviocites (Ogura et al. 2010), or $\mathrm{TNF} \alpha$ (Constantin et al. 2008) which is able to induce the synthesis of CCL2 in fibroblasts from colorectal liver metastases (Mueller et al. 2010). In any case, our results demonstrate that while CCL2 levels increase about eight times when NCTC 2472 cells are kept in culture, a higher increase of CCL2 levels (of about 12 times) is detected in the tumoral tissue, where fibrosarcoma cells grow in the vicinity of bone and immune cells. This augmentation in CCL2 levels is similar to that described after the calcaneus administration of these cells (Khasabova et al. 2007). Apart from increasing at tumoral level, CCL2 could also be upregulated at DRG cells, as described in neuropathic (Tanaka et al. 2004; White et al. 2005; Jung et al. 2008) or inflammatory settings (Jeon et al. 2008). However, no increase in CCL 2 levels was observed in DRG ipsilateral to the tibia inoculated with NCTC 2472 cells, representing this fact a difference with the above mentioned models. Thus, it may be concluded that in this model of bone cancer, sensitive neurons do not contribute to the augmentation of peripheral CCL2 and that its main source comes from the interplay between bone, tumoral, and immune cells.

In order to test whether the augmented presence of CCL2 measured in mice inoculated with NCTC 2472 cells could induce hyperalgesic responses, the effect of RS 5044393, a selective antagonist of CCR2 (Mirzadegan et al. 2000), was tested. The fact that both systemic and peritumoral administration of RS 504393 dose-dependently antagonized osteosarcoma-evoked thermal and mechanical hyperalgesia demonstrates that CCL2 is acting as hyperalgesic at tumoral level. Although the possibility that CCL2 can evoke hyperalgesic effects at spinal cord cannot be discarded, the complete inhibition of thermal and mechanical hyperalgesia obtained when CCR2 receptors are blocked at tumoral level demonstrates the involvement of peripheral CCR2 in neoplastic 
hypernociception. Furthermore, the antihyperalgesic effect obtained after the peritumoral administration of a mouse antibody against CCL2 chemokine strongly suggests that this endogenous chemokine could be responsible for this peripheral CCR2-mediated hyperalgesia.

In contrast with the antihyperalgesic effects obtained by blocking CCR2 receptors, mechanical allodynia due to tumoral development remained unaffected after CCR2 inhibition, thus indicating that the efficacy of an analgesic drug to inhibit distinct pain symptoms can be different. In this sense, previous studies from our laboratory described that when opioid or cannabinoid receptors are activated exclusively at the periphery, these drugs evoke antihyperalgesic, but not antiallodynic responses (Curto-Reyes et al. 2008; Curto-Reyes et al. 2010). The fact that peripheral mechanisms are more effective to inhibit hyperalgesia than allodynia both in the mentioned cases and in the present experiments seems to reflect the more important involvement of spinal mechanisms, such as central sensitisation, in the establishment of allodynia (Kim et al. 2012).

In order to explore if the involvement of peripheral CCL2 could be a general property of bone cancer pain, we have performed experiments with mice receiving the intratibial inoculation of B16-F10 melanoma cells. Although in clinical settings, bone metastases are not frequently associated to melanoma in comparison with prostate or breast tumors, the use of this experimental model offers the possibility of exploring the pharmacological properties of analgesic drugs when the underlying bone modifications due to the presence of cancer cells are different than those of the osteosarcoma model (Curto-Reyes et al. 2008). Previous data related to the release of CCL2 from human melanoma tumoral cells (Li et al. 2009) suggested the possibility that B16-F10 cells could release CCL2. However, demonstrating that the release of CCL2 is not a general property of tumoral cells able to evoke bone painful tumors, B16-F10 cells did not produce this chemokine in culture or after intratibial inoculation. In agreement with the lack of increased CCL2 tumoral levels in mice inoculated with B16-F10 cells, RS 504393 was almost ineffective against thermal hyperalgesia and completely inactive against mechanical hyperalgesia and allodynia. Although the fact that B16-F10 cells do not induce an increase in CCL2 levels in the tumoral paw could suggest that the reactivity to nociceptive stimuli in these melanoma-bearing mice should be lower than that measured in NCTC 2472-inoculated mice, a similar degree of thermal and mechanical hypernociception appears in mice inoculated either with NCTC 2472 or B16F10 cells (Baamonde et al. 2007; Curto-Reyes et al. 2008).

Globally, the present results demonstrate that the peripheral release of CCL2 is involved in thermal and mechanical hyperalgesia, but not mechanical allodynia evoked by the intraosteal inoculation of NCTC 2472 cells, whereas hypernociceptive symptoms produced after the inoculation of B16-F10 melanoma cells are CCL2-independent. This indicates that CCL2 is not a hyperalgesic mediator involved in all types of painful tumors and suggests that CCR2 antagonists could be useful to reduce hyperalgesia only in the particular type of tumors in which CCL2 is involved.

Acknowledgments Grants were provided by MICINN-FEDER (SAF2009-10567). M.P. is a recipient of a grant from the Instituto Universitario de Oncología (IUOPA). IUOPA is supported by Obra Social Cajastur-Asturias, Spain.

\section{References}

Abbadie C, Lindia JA, Cumiskey AM, Peterson LB, Mudgett JS, Bayne EK, DeMartino JA, MacIntyre DE, Forrest MJ (2003) Impaired neuropathic pain responses in mice lacking the chemokine receptor CCR2. Proc Natl Acad Sci USA 100:7947-7952

Abbadie C, Bhangoo S, De Koninck Y, Malcangio M, MelikParsadaniantz S, White FA (2009) Chemokines and pain mechanisms. Brain Res Rev 60:125-134

Baamonde A, Curto-Reyes V, Juárez L, Meana A, Hidalgo A, Menéndez L (2007) Antihyperalgesic effects induced by the IL-1 receptor antagonist anakinra and increased IL-1beta levels in inflamed and osteosarcoma-bearing mice. Life Sci 81:673-682

Baamonde A, Hidalgo A, Menéndez L (2011) Involvement of glutamate NMDA and AMPA receptors, glial cells and IL-1 $\beta$ in the spinal hyperalgesia evoked by the chemokine CCL2 in mice. Neurosci Lett 502:178-181

Belkouch M, Dansereau MA, Réaux-Le Goazigo A, Van Steenwinckel J, Beaudet N, Chraibi A, Melik-Parsadaniantz S, Sarret P (2011) The chemokine CCL2 increases Nav1.8 sodium channel activity in primary sensory neurons through a $\mathrm{G} \beta \gamma$-dependent mechanism. J Neurosci 31:18381-18390

Bogen O, Dina OA, Gear RW, Levine JD (2009) Dependence of monocyte chemoattractant protein 1 induced hyperalgesia on the isolectin B4-binding protein versican. Neuroscience 159:780-786

Chaplan SR, Bach FW, Pogrel JW, Chung JM, Yaksh TL (1994) Quantitative assessment of tactile allodynia in the rat paw. J Neurosci Meth 53:55-63

Chehl N, Gong Q, Chipitsyna G, Aziz T, Yeo CJ, Arafat HA (2009) Angiotensin II regulates the expression of monocyte chemoattractant protein-1 in pancreatic cancer cells. J Gastrointest Surg 13:2189-2200

Constantin CE, Mair N, Sailer CA, Andratsch M, Xu ZZ, Blumer MJ, Scherbakov N, Davis JB, Bluethmann H, Ji RR, Kress M (2008) Endogenous tumor necrosis factor alpha (TNFalpha) requires TNF receptor type 2 to generate heat hyperalgesia in a mouse cancer model. J Neurosci 28:5072-5081

Curto-Reyes V, Juárez L, García-Pérez E, Fresno MF, Hidalgo A, Menéndez L, Baamonde A (2008) Local loperamide inhibits thermal hyperalgesia but not mechanical allodynia induced by intratibial inoculation of melanoma cells in mice. Cell Mol Neurobiol 28:981-990

Curto-Reyes V, Llames S, Hidalgo A, Menéndez L, Baamonde A (2010) Spinal and peripheral analgesic effects of the CB2 cannabinoid receptor agonist AM1241 in two models of bone cancerinduced pain. Br J Pharmacol 160:561-573

Dansereau MA, Gosselin RD, Pohl M, Pommier B, Mechighel P, Mauborgne A, Rostene W, Kitabgi P, Beaudet N, Sarret P, Melik-Parsadaniantz S (2008) Spinal CCL2 pronociceptive action is no longer effective in CCR2 receptor antagonist-treated rats. J Neurochem 106:757-769 
Gao YJ, Zhang L, Samad OA, Suter MR, Yasuhiko K, Xu ZZ, Park JY, Lind AL, Ma Q, Ji RR (2009) JNK-induced MCP-1 production in spinal cord astrocytes contributes to central sensitization and neuropathic pain. J Neurosci 29:4096-4108

Jeon SM, Lee KM, Park ES, Jeon YH, Cho HJ (2008) Monocyte chemoattractant protein-1 immunoreactivity in sensory ganglia and hindpaw after adjuvant injection. Neuroreport 19:183-186

Jeon SM, Sung JK, Cho HJ (2011) Expression of monocyte chemoattractant protein-1 and its induction by tumor necrosis factor receptor 1 in sensory neurons in the ventral rhizotomy model of neuropathic pain. Neuroscience 190:354-366

Jung H, Toth PT, White FA, Miller RJ (2008) Monocyte chemoattractant protein-1 functions as a neuromodulator in dorsal root ganglia neurons. J Neurochem 104:254-263

Khasabova IA, Stucky CL, Harding-Rose C, Eikmeier L, Beitz AJ, Coicou LG, Hanson AE, Simone DA, Seybold VS (2007) Chemical interactions between fibrosarcoma cancer cells and sensory neurons contribute to cancer pain. J Neurosci 27:10289-10298

Kim YH, Back SK, Davies AJ, Jeong H, Jo HJ, Chung G, Na HS, Bae YC, Kim SJ, Kim JS, Jung SJ, Oh SB (2012) TRPV1 in GABAergic interneurons mediates neuropathic mechanical allodynia and disinhibition of the nociceptive circuitry in the spinal cord. Neuron 74:640-647

Li L, Dragulev B, Zigrino P, Mauch C, Fox JW (2009) The invasive potential of human melanoma cell lines correlates with their ability to alter fibroblast gene expression in vitro and the stromal microenvironment in vivo. Int J Cancer 25:1796-1804

Menéndez L, Lastra A, Fresno MF, Llames S, Meana A, Hidalgo A, Baamonde A (2003) Initial thermal heat hypoalgesia and delayed hyperalgesia in a murine model of bone cancer pain. Brain Res 969:102-109

Menéndez L, Lastra A, Meana A, Hidalgo A, Baamonde A (2005) Analgesic effects of loperamide in bone cancer pain in mice. Pharmacol Biochem Behav 81:114-121

Mirzadegan T, Diehl F, Ebi B, Bhakta S, Polsky I, McCarley D, Mulkins M, Weatherhead GS, Lapierre JM, Dankwardt J, Morgans D Jr, Wilhelm R, Jarnagin K (2000) Identification of the binding site for a novel class of CCR2b chemokine receptor antagonists. J Biol Chem 275:25562-25571

Mueller L, von Seggern L, Schumacher J, Goumas F, Wilms C, Braun F, Broering DC (2010) TNF-alpha similarly induces IL-6 and MCP-1 in fibroblasts from colorectal liver metastases and normal liver fibroblasts. Biochem Biophys Res Commun 397:586-591
Ogura N, Satoh K, Akutsu M, Tobe M, Kuyama K, Kuboyama N, Sakamaki H, Kujiraoka H, Kondoh T (2010) MCP-1 production in temporomandibular joint inflammation. J Dent Res 89:1117-1122

Owen JL, Torroella-Kouri M, Handel-Fernandez ME, IragavarapuCharyulu V (2007) GM-CSF upregulates the expression of CCL 2 by $\mathrm{T}$ lymphocytes in mammary tumor-bearing mice. Int $\mathrm{J}$ Mol Med 20:129-136

Qin X, Wan Y, Wang X (2005) CCL2 and CXCL1 trigger calcitonin gene-related peptide release by exciting primary nociceptive neurons. J Neurosci Res 82:51-62

Schiller KR, Zillhardt MR, Alley J, Borjesson DL, Beitz AJ, Mauro LJ (2009) Secretion of MCP-1 and other paracrine factors in a novel tumor-bone coculture model. BMC Cancer 9:45

Schweizerhof M, Stösser S, Kurejova M, Njoo C, Gangadharan V, Agarwal N, Schmelz M, Bali KK, Michalski CW, Brugger S, Dickenson A, Simone DA, Kuner R (2009) Hematopoietic colony-stimulating factors mediate tumor-nerve interactions and bone cancer pain. Nat Med 15:802-807

Shirotake S, Miyajima A, Kosaka T, Tanaka N, Kikuchi E, Mikami S, Okada Y, Oya M (2012) Regulation of monocyte chemoattractant protein-1 through angiotensin II type 1 receptor in prostate cancer. Am J Pathol 180:1008-1016

Soria G, Ofri-Shahak M, Haas I, Yaal-Hahoshen N, Leider-Trejo L, Leibovich-Rivkin T, Weitzenfeld P, Meshel T, Shabtai E, Gutman $\mathrm{M}$, Ben-Baruch A (2011) Inflammatory mediators in breast cancer: coordinated expression of TNF $\alpha$ \& IL-1 $\beta$ with CCL2 \& CCL5 and effects on epithelial-to-mesenchymal transition. BMC Cancer 11:130

Stösser S, Schweizerhof M, Kuner R (2011) Hematopoietic colonystimulating factors: new players in tumor-nerve interactions. J Mol Med (Berl) 89:321-329

Tanaka T, Minami M, Nakagawa T, Satoh M (2004) Enhanced production of monocyte chemoattractant protein-1 in the dorsal root ganglia in a rat model of neuropathic pain: possible involvement in the development of neuropathic pain. Neurosci Res 48:463-469

Wang XM, Hamza M, Wu TX, Dionne RA (2009) Upregulation of IL6, IL-8 and CCL2 gene expression after acute inflammation: correlation to clinical pain. Pain 142:275-283

White FA, Bhangoo SK, Miller RJ (2005) Chemokines: integrators of pain and inflammation. Nat Rev Drug Discov 4:834-844

Zimmermann M (1983) Ethical guidelines for investigations of experimental pain in conscious animals. Pain 16:109-110 